\title{
Strong magnetoelastic coupling in VOCl: Neutron and synchrotron powder x-ray diffraction study
}

\author{
A. C. Komarek, ${ }^{1}$ T. Taetz, ${ }^{2}$ M. T. Fernández-Díaz, ${ }^{3}$ D. M. Trots, ${ }^{4}$ A. Möller, ${ }^{2}$ and M. Braden ${ }^{1, *}$ \\ ${ }^{1}$ II. Physikalisches Institut, Universität zu Köln, Zülpicher Str. 77, D-50937 Köln, Germany \\ ${ }^{2}$ Institut für Anorganische Chemie, Universität zu Köln, Greinstr. 6, D-50939 Köln, Germany \\ ${ }^{3}$ Institut Laue-Langevin, 38042 Grenoble, France \\ ${ }^{4}$ Hasylab/DESY, Notkestr. 85, D-22607, Hamburg, Germany \\ (Received 10 November 2008; revised manuscript received 3 February 2009; published 24 March 2009)
}

\begin{abstract}
We present a combined neutron and synchrotron powder-diffraction study of the crystal and magnetic structures of $\mathrm{VOCl}$. The occurrence of antiferromagnetic order in $\mathrm{VOCl}$ is accompanied by a monoclinic distortion. The high sensitivity of the magnetic interaction parameters on $\mathrm{V}-\mathrm{O}-\mathrm{V}$ bond angles and $\mathrm{V}-\mathrm{V}$ distances yields strong magnetoelastic coupling which implies the structural distortion below $T_{N}$ as well as anomalous structural effects in the paramagnetic phase.
\end{abstract}

DOI: 10.1103/PhysRevB.79.104425

PACS number(s): 75.25.+z, 75.30.Kz, 75.50.-y

\section{INTRODUCTION}

The transition-metal oxyhalides $M \mathrm{O} X$, with $M=\mathrm{Sc}, \mathrm{Ti}, \mathrm{V}$, $\mathrm{Cr}, \mathrm{Fe}$ and $X=\mathrm{Cl}, \mathrm{Br}$, have attracted strong interest in the past years initiated through the challenging low-dimensional phenomena observed for TiOCl. ${ }^{1-6}$ At $T_{1}=67 \mathrm{~K}$, TiOCl shows a transition associated with a dimerization of the $\mathrm{Ti}$ ions along the $b$ direction (first-order phase transition) which has been interpreted as a spin Peierls transition. Thus, TiOCl might be a rare case of an inorganic spin Peierls system similar to $\mathrm{CuGeO}_{3} \cdot{ }^{1,2,7}$ The whole group of transition-metal oxyhalides $M O X$ provides an interesting class of-at room temperature-isostructural compounds in which several electronic configurations and their interactions with the crystal lattice can be studied systematically. However, the physical properties of the $\mathrm{MOCl}$ compounds different from $\mathrm{TiOCl}$ have been studied in less detail so far. ${ }^{8-12}$ Like TiOCl, VOCl crystallizes in an orthorhombic structure with space group Pmmn, with a single crystallographic V site. ${ }^{13}$ The $\mathrm{V}^{3+}$ ions are coordinated by four oxygen atoms and two chlorine atoms forming strongly distorted $\mathrm{VO}_{4} \mathrm{Cl}_{2}$ octahedrons (see Fig. 1). These are linked via corners and edges forming quasitwo-dimensional bilayers in the $a b$ plane. Due to the $\mathrm{Cl}$ - ions there is a clear separation of neighboring Ti-O bilayers explaining the electronic and magnetic low-dimensional characters. Due to electronic correlations all oxyhalides are Mott insulators. In $\mathrm{VOCl}$ the band gap is reported to be about 1.7 eV. ${ }^{14}$ Whereas a direct overlap of the ground state $y z$ orbitals (with reference to the cell axis $b$ and $c$ ) along the crystallographic $b$ axis leads to one-dimensional magnetic interactions in $\mathrm{TiOCl}^{4}{ }^{\mathrm{VOCl}}$ is believed to exhibit quasi-twodimensional antiferromagnetic ordering below $T_{N}(80 \mathrm{~K}) .{ }^{9} \mathrm{In}$ an early powder neutron diffraction study Wiedenman et al. ${ }^{9}$ reported a collinear magnetic order with the wave vector $(1 / 2$ $1 / 21 / 2$ ) and the magnetic moments pointing in $a$ direction. The magnetic susceptibility was well described within a quadratic two-dimensional (2D)-Heisenberg model. However, no signs of a structural transition in $\mathrm{VOCl}$ have been observed at low temperatures.

In this work, we report $\mathrm{x}$-ray and neutron powderdiffraction experiments on $\mathrm{VOCl}$ which reveal a structural phase transition accompanying the antiferromagnetic order in VOCl. We find a remarkably strong magnetoelastic coupling in $\mathrm{VOCl}$ which seems to arise from the dependence of the magnetic interaction on soft lattice degrees of freedom, $\mathrm{V}-\mathrm{O}-\mathrm{V}$ bond angles, and $\mathrm{V}-\mathrm{V}$ distances, in analogy to the spin-Peierls compound $\mathrm{CuGeO}_{3}$. ${ }^{7,15,16}$

\section{EXPERIMENTAL}

$\mathrm{VOCl}$ was prepared by the chemical transport technique as described by Schäfer and Wartenpfuhl. ${ }^{17}$ Single crystals obtained via this route were thoroughly ground, compressed under hydrostatic pressure, and reground afterwards. This procedure was repeated several times in order to achieve a homogenous powder and hence minimize preferred orientation effects. The phase purity of the sample was checked several times using powder $\mathrm{x}$-ray diffraction.
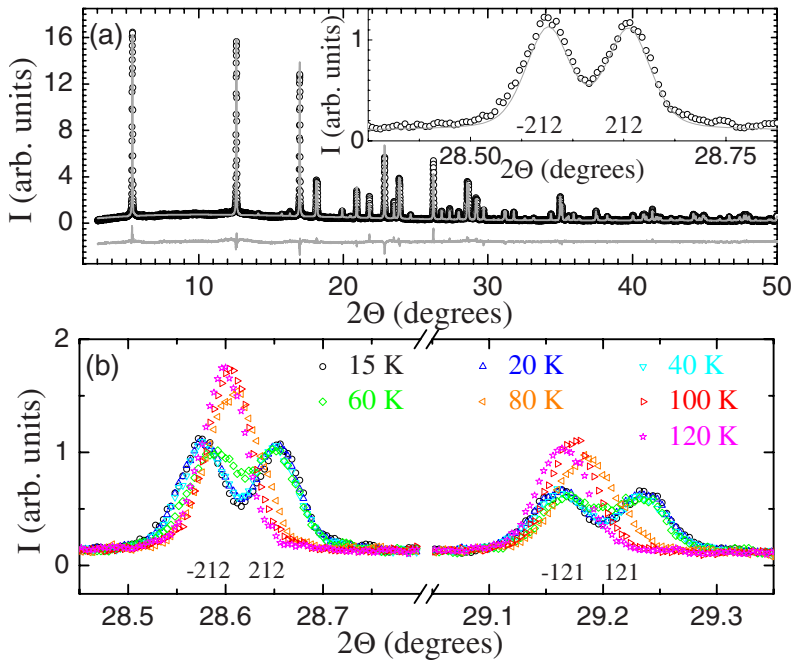

FIG. 1. (Color online) Synchrotron-radiation powder X-ray diffraction patterns. (a) Rietveld fit (gray) to the data at $15 \mathrm{~K}$ (black) with an enlargement of the diffraction pattern around the $( \pm 212)$ profiles in the inset. (b) Temperature dependency of the $( \pm 212)$ and $( \pm 121)$ profiles. 

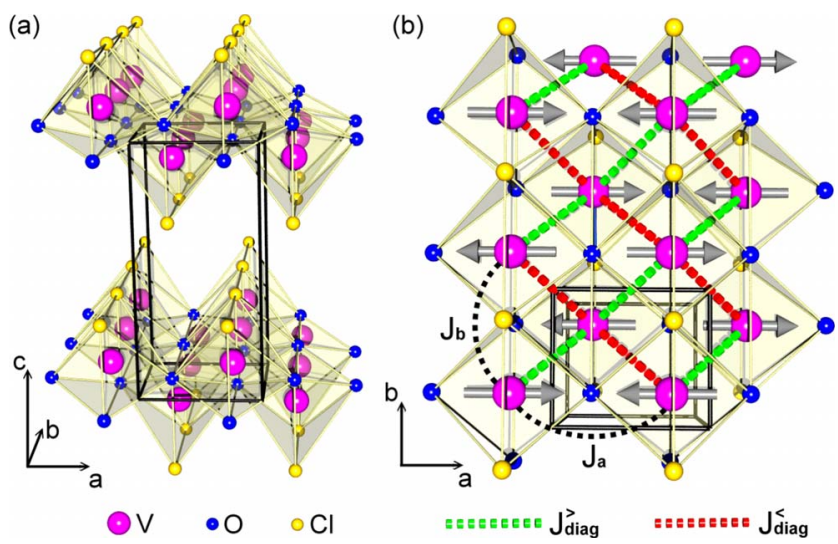

FIG. 2. (Color online) Nuclear and magnetic structures of VOCl. Yellow: $\mathrm{Cl}$, blue: $\mathrm{O}$, magenta: V ions, black: the structural unit cell. The directions of the magnetic moments at each $\mathrm{V}$ site is indicated by magenta arrows. Green (red) dotted lines indicate the antiferromagnetically (ferromagnetically) coupled V-V pairs in the AFM1 structure.

Synchrotron-radiation powder x-ray diffraction measurements have been performed at beamline B2 at Hasylab/ DESY in Hamburg, Germany. ${ }^{18}$ The single phase VOCl powder was finely ground and filled in a glass capillary with 0.3 $\mathrm{mm}$ in diameter. The incident $\mathrm{x}$-ray wavelength was $0.7466 \AA$ and the reflections have been measured using an image-plate detector in Debye-Scherrer geometry. ${ }^{19}$

Powder neutron-diffraction measurements have been performed at the D1A, D2B, and D20 diffractometers at the ILL in Grenoble, France $(\lambda=1.91,1.594$, and $2.4233 \AA$, respectively). The samples measured at the D20 and D2B diffractometers contained some unidentified impurity phases. Therefore, only the single phase sample measured at the D1A diffractometer was used for a full nuclear-structure refinement. The high-intensity D20 data have been used for the magnetic structure refinement, as there was no overlap of peaks from the impurity phases with the magnetic peaks due to the longer wavelength.

\section{RESULTS AND DISCUSSION}

\section{A. Monoclinic distortion}

The temperature-dependent powder $\mathrm{x}$-ray diffraction measurements using synchrotron radiation clearly reveal a structural phase transition below $80 \mathrm{~K}$. A diffraction pattern at the lowest temperature, $15 \mathrm{~K}$, is shown in Fig. 2(a). The open circles denote the measured data points and the line indicates the Rietveld fit of the structure model using the program FULLPROF/WINPLOTR. ${ }^{20}$ The part of the diffraction pattern around the $( \pm 212)$ reflections is shown in the inset. The clearly observable splitting of the orthorhombic (212) reflection indicates the symmetry reduction from the orthorhombic (Pmmn) to a monoclinic symmetry. The data can be well described in space group $P 2 / n$ (monoclinic setting $c$ ) and a monoclinic angle $\gamma$ of $90.2^{\circ}$. In Fig. 2(b) the $( \pm 212)$ and $( \pm 121)$ reflections are plotted for different temperatures indicating the orthorhombic to monoclinic phase transition below $80 \mathrm{~K}$. The resulting lattice parameters are plotted as a

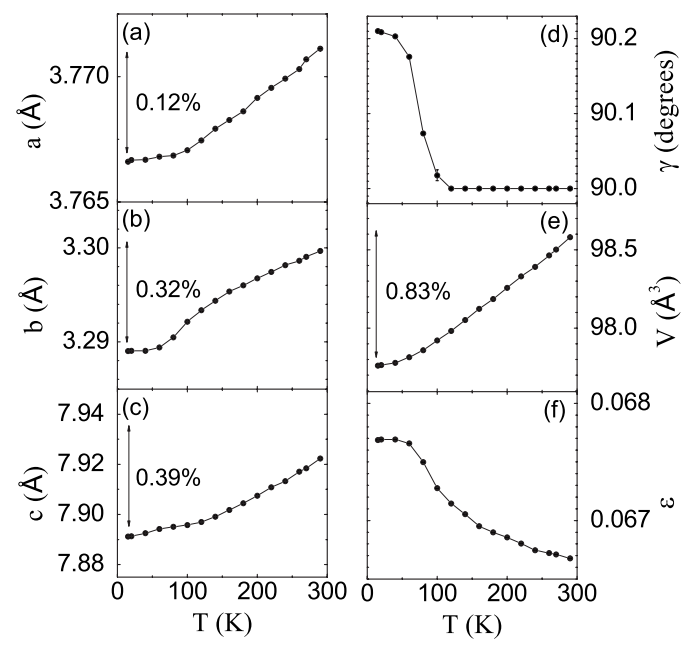

FIG. 3. Results of synchrotron-radiation powder x-ray diffraction measurements: [(a)-(c)] lattice parameter, (d) monoclinic angle, (e) unit-cell volume, and (f) orthorhombic splitting $\varepsilon=(a$ $-b) /(a+b)$. (Lines are guide to the eyes.)

function of temperature in Figs. 3(a)-3(d). A rise of the monoclinic angle $\gamma$ can be observed below $80 \mathrm{~K}$ reaching its maximum value of about $90.2^{\circ}$ around $40 \mathrm{~K}$. The monoclinic distortion and the structural phase transition apparently have escaped detection in the previous neutron-diffraction experiments due to their limited resolution. ${ }^{9}$ The group-subgroup relationship between Pmmn and $P 2 / n$ as well as the small change in the unit-cell volume suggest a second-order phase transition.

Powder neutron-diffraction measurements have been performed at the ILL. In Figs. 4(a) and 4(b) neutron-diffraction patterns of $\mathrm{VOCl}$ are shown. Figure 4(a) shows the magnetic peaks measured at $2 \mathrm{~K}$ at the high-flux D20 diffractometer
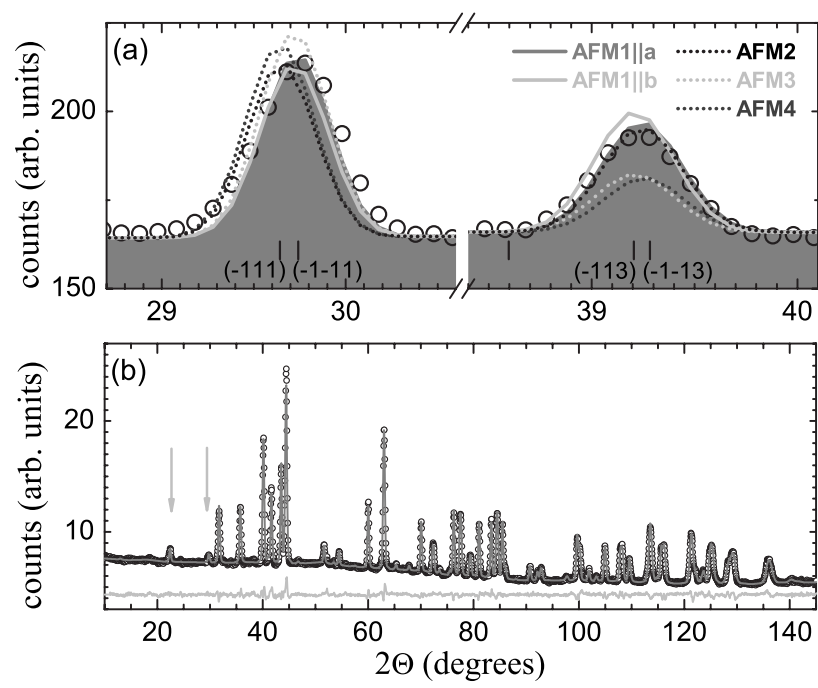

FIG. 4. (a) Powder neutron-diffraction patterns measured at $2 \mathrm{~K}$ (D20 diffractometer). Circles: data points, lines: Rietveld fits to the data with the AFM1-AFM4 structure models. The area below the AFM1 fit (moment $\| a$ ) is filled (gray). (b) Fit of the D1A measurement at $10 \mathrm{~K}$; black: data points, gray: Rietveld fit to the data, light gray: difference $\mathrm{I}_{\mathrm{obs}}-\mathrm{I}_{\text {calc }}$, arrows indicate the magnetic peaks. 
with an incident wavelength of $2.4233 \AA$. In Fig. 4(b) an entire diffractogram measured at $10 \mathrm{~K}$ on the D1A diffractometer using a neutron wavelength of $1.91 \AA$ and higher resolution is shown. The latter data were used for structural refinements.

The appearance of magnetic peaks at (1/2 1/2 1/2) and $(1 / 21 / 23 / 2)$ with respect to the nuclear lattice clearly indicates the antiferromagnetic order occurring below $T_{N}$ $\sim 80 \mathrm{~K}$ in perfect agreement with Ref. 9. A fully threedimensional (3D) antiferromagnetic structure could be fitted to the neutron data [see Fig. 4(a)]. The half-indexed reflections indicate the magnetic propagation vector $\mathbf{k}$ $=(1 / 21 / 21 / 2)$ corresponding to a $2 \times 2 \times 2$ enlargement of the magnetic unit cell.

Within the nuclear unit cell there are two magnetic sites: the $\mathrm{V}$ ions at $(0.75,0.25,0.117)$ and at $(0.25,0.75,-0.117)$ which belong to two different chains of edge-sharing $\mathrm{VO}_{4} \mathrm{Cl}_{2}$ octahedrons along the $b$ direction, labeled $b$ chains in the following. These $b$ chains form the VO planes parallel to the $a, b$ planes and are situated alternatingly above and below the $z=0$ level. The inspection of the magnetic paths in Figs. 1(a) and 1(b) allows one to qualitatively understand the antiferromagnetic order. There is a magnetic interaction $J_{a}$ along $a$ mediated through the common $\mathrm{O}$ between two $\mathrm{V}$ sites at the same $z$ level. This antiferromagnetic interaction, which is absent in $\mathrm{TiOCl}$ due to the single occupation of the $d_{y z}$ orbital, only acts on $\mathrm{V}$ ions in next-nearest-neighbor $b$ chains yielding an antiferromagnetic arrangement between these next-nearest $b$ chains and thus a doubling of the $a$ parameter. There is also an interaction $J_{b}$ along $b$ mediated through the common octahedron edges which results in an antiferromagnetic order within the $b$ chains and doubling along $b$. With an additional weak antiferromagnetic coupling perpendicular to the planes one may thus explain the doubling of the magnetic lattice along the three orthorhomic directions. However, in the orthorhombic phase the interaction between the nearest-neighbor $b$ chains is fully frustrated since the path parallel to [110] and that parallel to [1-10] are equivalent by symmetry and thus $J_{\mathrm{diag}}^{>}=J_{\mathrm{diag}}^{<}$. Since these two interaction parameters connect to spins of opposite signs in the nearest-neighbor $b$ chain the coupling is fully frustrated in the orthorhombic phase.

The two possible magnetic arrangements in the orthorhombic phase, however, do not correspond to two distinct magnetic symmetries but only to two domains of equivalent magnetic symmetry. This conclusion is fully corroborated by the representation analysis of the orthorhombic structure which only yields one irreducible representation for the propagation vector $\mathbf{k}=(1 / 21 / 21 / 2)$ and magnetic moments aligned parallel to the $a, b$ planes. The monoclinic distortion accompanying the magnetic order lifts the frustration of the nearest-neighbor $b$-chain coupling, $J_{\text {diag }}^{>} \neq J_{\text {diag }}^{<}$, since it renders the magnetic paths parallel to [110] and [1-10] inequivalent. As indicated in Fig. 1(b), all the $J_{\text {diag }}$ paths parallel to [110], $J_{\text {diag }}^{>}$, are equivalent in the monoclinic phase but they differ from the $J_{\text {diag }}$ paths parallel to [1-10], $J_{\text {diag }}^{<}$. Supposing that $J_{\text {diag }}^{>}$is larger than $J_{\text {diag }}^{<}$the magnetic ordering shown in Fig. 1(b) is stabilized. We may thus conclude that the monoclinic distortion accompanying the magnetic transition lifts the frustration of the magnetic coupling between nearest-neighbor $b$ chains.
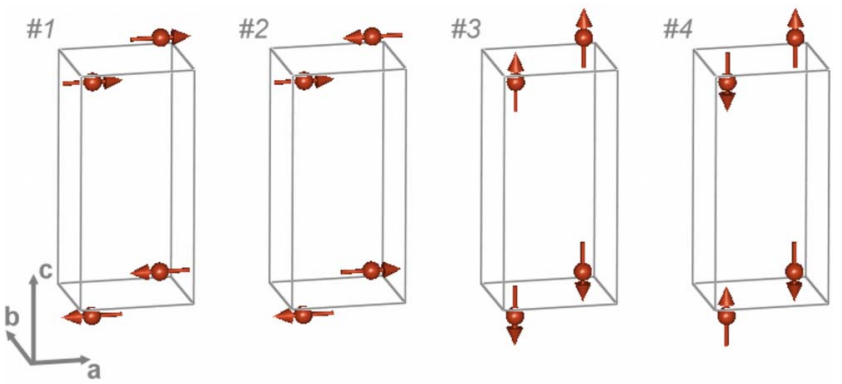

FIG. 5. (Color online) (a)-(d) The possible irreducible representations in space group $P 112 / n$ for $k=\left(\begin{array}{lll}1 / 2 & 1 / 2 & 1 / 2\end{array}\right)$ with magnetic atoms in the primitive unit cell at $\left(-0.25,0.25, z_{0}\right)$. For (a) and (b) the presented spin direction along $a$ indicates the direction of the ordered moment found in $\mathrm{VOCl}$, which exhibits magnetic order according to the representation 1 .

\section{B. Magnetic structure}

Let us now turn to the quantitative analysis of the magnetic structure in $\mathrm{VOCl}$. The two strong magnetic Bragg peaks observed at $(1 / 21 / 21 / 2)$ and $(1 / 21 / 23 / 2)$ are best described by the model with ferromagnetic coupling between the two $\mathrm{V}$ moments at $(0.75,0.25,+z)$ and $(0.25,0.75,-z)$ pointing in $a$ direction (see Fig. 1). Other directions of the magnetic moment such as the [110] direction induce discrepancies in the measured intensities of the $( \pm 1 \pm 11)$ and $( \pm 1 \pm 13)$ reflections (in notation of the magnetic unit cell).

Due to representation theory, there are four irreducible representations for space group $P 112 / n$ with a magnetic ion at $\left(\begin{array}{lll}-0.25 & 0.25 & z_{0}\end{array}\right)\left(z_{0} \approx 0.117\right)$ and with propagation vector $\vec{k}=\left(\begin{array}{lll}1 / 2 & 1 / 2 & 1 / 2\end{array}\right)$. The basis vectors for these four possibilities derived from the program BASIREP (Ref. 20) are shown in Fig. 5. These four possibilities are referred to as the antiferromagnetic structures AFM1 to AFM4. The representation analysis separates the arrangements with the moment parallel to $c$ (AFM3 and AFM4) from those with in-plane moments (AFM1 and AFM2). For these two cases, there is the possibility of ferromagnetic or antiferromagnetic coupling of the two sites at $(0.75,0.25,+z)$ and $(0.25,0.75,-z)$. The two magnetic structures AFM1 and AFM2 (or AFM3 and AFM4) cannot be distinguished if the crystal structure is orthorhombic (Pmmn). However, the structural phase transition $P m m n \rightarrow P 2 / n$ observed in our work induces a splitting of the $( \pm 1 \mp 11)$ and the $( \pm 1 \pm 13)$ reflections (referring to the magnetic cell). The small splitting of these reflections allows us to distinguish between the two magnetic structures. In Fig. 4(a) the Rietveld fits to the measured data at $2 \mathrm{~K}$ are shown for the four magnetic models. In the Rietveld fit of the AFM2 structure, the calculated intensities for the first magnetic peak $( \pm 1 \pm 11)$ are shifted to lower $2 \Theta$ values in contradiction to the experiment. In contrast, the AFM1 structure perfectly describes the data if the magnetic moments are parallel to the $a$ direction. A fit with moments in $b$ direction does not result in a correct description of the intensities. Even the free refinement of both $a$ and $b$ components yields only negligible values for the moment in $b$ direction. This observation of the ordered moment along the $a$ direction is in accordance with the measured magnetic susceptibility in Ref. 9 which sharply 

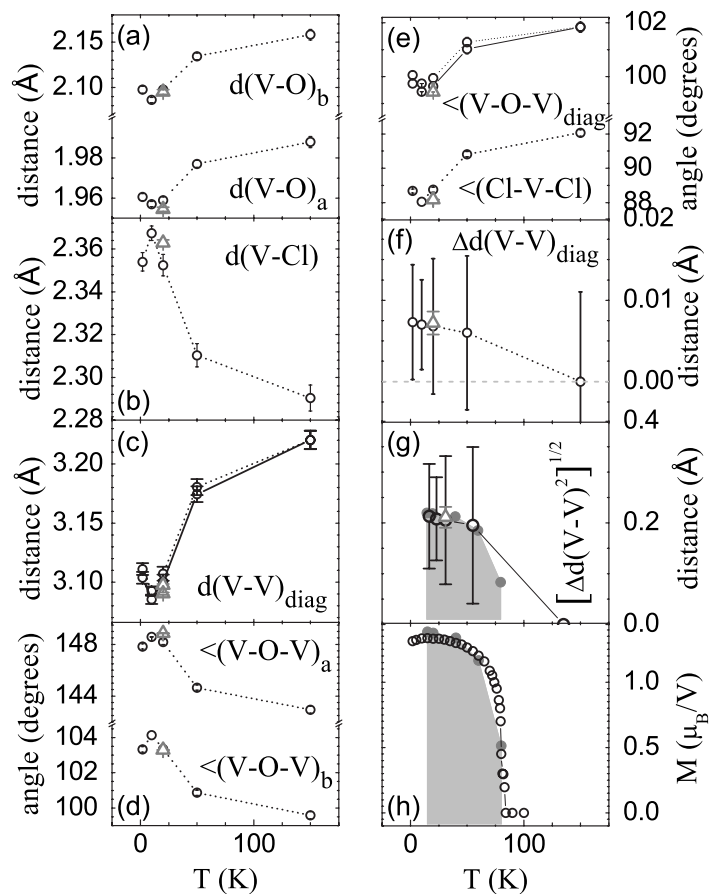

FIG. 6. Results of powder neutron diffraction (black circles) and synchrotron measurements (gray triangles). (a) $\mathrm{V}-\mathrm{O}$ distances for bonds in $a$ and $b$ directions. (b) $\mathrm{V}-\mathrm{Cl}$ distance. (c) $\mathrm{V}-\mathrm{V}$ distances for $\mathrm{V}$ ions within the same $\mathrm{V}$ bilayer in diagonal direction (red and green bonds in Fig. 1). (d) V-O-V bond angles for $\mathrm{V}$ ions (with the same value of $z$ ) in $a$ direction $(\mathrm{V}-\mathrm{O}-\mathrm{V})_{a}$ and in $b$ direction $(\mathrm{V}-\mathrm{O}-\mathrm{V})_{b}$. (e) $\mathrm{Cl}-\mathrm{V}-\mathrm{Cl}$ angle and $\mathrm{V}-\mathrm{O}-\mathrm{V}$ angle in diagonal direction. (f) $\Delta d(\mathrm{~V}-\mathrm{V})_{\text {diag }}$ denotes the difference of the two diagonal $\mathrm{V}-\mathrm{V}$ distances. (g) $\sqrt{\left\{d(V-V)_{\text {diag, } 1}\right\}^{2}-\left\{d(V-V)_{\text {diag, } 2\}^{2}}\right.}$ plotted with the monoclinic angle $\gamma$ (gray). (h) The antiferromagnetic moment (black) together with $\gamma$ (gray). (Lines are guide to the eyes.)

drops along the $a$ direction below $T_{N}$. The AFM3 and AFM4 structures with $c$ components do not properly describe the magnetic intensities [see Fig. 4(a)]. In VOCl the AFM1 magnetic structure with moments in $a$ direction is thus realized. In Ref. 9, a mixture of the AFM1 and AFM2 was proposed basing on single-crystal diffraction, however, most likely, the twinning induced by the structural phase transition, which was not detected in that work, occurs in the used single crystal fully explaining the observed intensities.

The antiferromagnetic moment amounts to about $1.3 \mu_{B}$ at $2 \mathrm{~K}$ which is in approximate agreement with the value reported in Ref. 9 but which is smaller than the full spin moment expected for the vanadium ion with a $3 d^{2}$ electronic configuration. The value of the antiferromagnetic moment as a function of temperature is shown in Fig. 6(h). The temperature dependency of this antiferromagnetic moment indicates a Néel temperature of $T_{N} \sim 80 \mathrm{~K}$.

\section{Magnetoelastic coupling in VOCl}

The structural refinements reveal a displacement of the vanadium ions away from the chlorine toward the oxygen sites in $c$ direction upon cooling [see Figs. 6(a)-6(d)]. The VO planes become thus flatter at low temperature. In conse- quence the $\mathrm{V}-\mathrm{O}-\mathrm{V}$ bond angle along $a$ increases upon cooling thereby enhancing the antiferromagnetic exchange interaction $J_{a}$. The V-O-V path along $a$ exhibits a bond angle considerably smaller than $180^{\circ}$ but larger than the value of $90^{\circ}$ where one expects the antiferromagnetic superexchange to vanish. ${ }^{7,15,16}$ In consequence the magnetic interaction $J_{a}$ depends sensitively on the bond angle and increases with its enhancement. The direct overlap of the $\mathrm{V}$ orbitals along $b$ seems to dominate the magnetic interaction $J_{b}$ which increases upon cooling due to the shrinking of the $b$ lattice parameter. The structural changes implied through the cooling appear thus to be driven by the magnetism which favors an enhancement of the magnetic interaction parameters at low temperature.

Magnetoelastic coupling is also evident in the coupling of the magnetic transition with the monoclinic distortion. In Fig. 6(h) we plot the ordered magnetic moment together with the monoclinic distortion as a function of temperature demonstrating the close coupling. The structural phase transition from Pmmn to $P 112 / n$ symmetry is of proper ferroelastic character, so that the monoclinic distortion is the primary structural order parameter.

More quantitatively, the preference of the AFM1 structure can be understood with regard to the monoclinic distortion of the structure. As noted above, the magnetic arrangement is antiferromagnetic along the V-V bonds, $J_{\text {diag }}^{>}$(green in Fig. 1 ), but ferromagnetic along $J_{\text {diag }}^{<}$(red). Unfortunately, the neutron measurements suffer from the small vanadium scattering length causing enlarged error bars for variables which depend on one or on two V-position parameters. This effect is most visible in Fig. 6(f) where the difference of the bond lengths of the diagonal $\mathrm{V}-\mathrm{V}$ bonds (denoted by the red and green lines in Fig. 1) is shown. Therefore, an extremely long measurement has been performed on the D1A diffractometer in order to increase the statistics and another synchrotronradiation experiment at $20 \mathrm{~K}$ has been performed at beamline B2 at Hasylab/DESY. In this synchrotron experiment several image-plate data collections with a total measuring time of more than $1 \mathrm{~h}$ have been summed up. The results of these precise experiments are plotted together with the other neutron data in Figs. 6(a)-6(g). The error bars of the synchrotron measurements are of similar dimensions as the symbol size. Especially, the value of the difference of the diagonal V-V distances shown in Fig. 6(f) has much smaller error bars and corroborates the neutron results. The structural parameters of this synchrotron measurement and of the neutron measurements at 2 and at $150 \mathrm{~K}$ are listed in Table I. The difference in the squares of the diagonal $\mathrm{V}-\mathrm{V}$ distances is determined with much higher precision as the $\mathrm{V} z$ parameter does not enter its calculation which only depends on the projection of the distances onto the $a, b$ planes [see Fig. $6(\mathrm{~g})$ ]. Since the $x$ and $y$ coordinates are fixed, the difference in the squares of the $\mathrm{V}-\mathrm{V}$ distances is directly calculated from the lattice parameters.

Indeed, the two diagonal V-V bonds (red/green in Fig. 1) differ by $0.0073(15) \AA$ as can be seen in Fig. 6(f). The splitting of the diagonal $\mathrm{V}-\mathrm{V}$ distance is just a consequence of the monoclinic angle and clearly shows up in $\sqrt{\left\{d(V-V)_{\text {diag, } 1}\right\}^{2}-\left\{d(V-V)_{\text {diag, } 2\}^{2}}\right.}$ [see Fig. 6(g)]. Compared to the orthorhombic structure where the diagonal $\mathrm{V}-\mathrm{V}$ bonds 
TABLE I. Results of the structural refinement of the neutron (D1A) and synchrotron (B2) measurements.

\begin{tabular}{|c|c|c|c|c|}
\hline $2 \mathrm{~K}$ & Neutron & D1A (ILL) & & \\
\hline SG & $\mathbf{a}(\AA)$ & $\mathbf{b}(\AA)$ & $\mathbf{c}(\AA)$ & $\gamma$ \\
\hline$P 112 / n$ & $3.76755(10)$ & $3.29045(9)$ & $7.89582(20)$ & $90.21(0)$ \\
\hline Atom & $\mathbf{x}$ & $\mathbf{y}$ & $\mathbf{z}$ & \\
\hline $\mathrm{V}$ & -0.25 & 0.25 & $0.11678(76)$ & \\
\hline $\mathrm{Cl}$ & -0.25 & 0.75 & $0.33000(4)$ & \\
\hline $\mathrm{O}$ & -0.25 & $\begin{array}{c}0.75 \\
-0.04800(6)\end{array}$ & & \\
\hline $10 \mathrm{~K}$ & Neutron & D1A (ILL) & & \\
\hline$S G$ & $\mathbf{a}(\AA)$ & $\mathbf{b}(\AA)$ & $\mathbf{c}(\AA)$ & $\gamma$ \\
\hline$P 112 / n$ & $3.76776(6)$ & $3.29076(5)$ & 7.896 11(13) & $90.202(1)$ \\
\hline Atom & $\mathbf{x}$ & $\mathbf{y}$ & $\mathbf{z}$ & \\
\hline $\mathrm{V}$ & -0.25 & 0.25 & $0.11478(59)$ & \\
\hline $\mathrm{Cl}$ & -0.25 & 0.75 & $0.33035(3)$ & \\
\hline $\mathrm{O}$ & -0.25 & 0.75 & $-0.04765(3)$ & \\
\hline $150 \mathrm{~K}$ & Neutron & D1A (ILL) & & \\
\hline$S G$ & $\mathbf{a}(\AA)$ & $\mathbf{b}(\AA)$ & $\mathbf{c}(\AA)$ & $\gamma$ \\
\hline PmmnS & $3.77001(10)$ & $3.29656(10)$ & $7.90713(25)$ & 90 \\
\hline Atom & $\mathbf{x}$ & $\mathbf{y}$ & $\mathbf{z}$ & \\
\hline $\mathrm{V}$ & -0.25 & 0.25 & $0.12804(111)$ & \\
\hline $\mathrm{Cl}$ & -0.25 & 0.75 & $0.32914(5)$ & \\
\hline $\mathrm{O}$ & -0.25 & 0.75 & $-0.04815(8)$ & \\
\hline $20 \mathrm{~K}$ & Synchrotron & B2 (DESY) & & \\
\hline$S G$ & $\mathbf{a}(\AA)$ & $\mathbf{b}(\AA)$ & $\mathbf{c}(\AA)$ & $\gamma$ \\
\hline$P 112 / n$ & $3.7654(3)$ & $3.2880(2)$ & $7.8894(5)$ & $90.207(1)$ \\
\hline Atom & $\mathbf{x}$ & $\mathbf{y}$ & $\mathbf{z}$ & \\
\hline $\mathrm{V}$ & -0.25 & 0.25 & $0.11555(15)$ & \\
\hline $\mathrm{Cl}$ & -0.25 & 0.75 & $0.33068(19)$ & \\
\hline $\mathrm{O}$ & -0.25 & 0.75 & $-0.04906(51)$ & \\
\hline
\end{tabular}

(red/green in Fig. 1) are equivalent, the monoclinic distortion renders the two antiferromagnetic exchange interactions inequivalent. We unambiguously find that the diagonal $\mathrm{V}-\mathrm{V}$ distance associated with $J_{\text {diag }}^{>}$is smaller than that associated with $J_{\text {diag }}^{<}$which indicates a stronger orbital overlap accompanied by a larger exchange interaction $J_{\text {diag }}^{>}$thereby explaining the observed magnetic structure. Since the magnetic energy gain due to the lifting of the frustration is linear in the structural distortion and since the lattice-energy cost scales quadratically with the distortion, one should always expect such an effect if the magnetic coupling between two subunits remains frustrated. However, in most cases, the magnetoelastic coupling would be too weak to generate an observable effect. The case of $\mathrm{VOCl}$ seems to profit from the fact that the diagonal interaction is modulated via the $\mathrm{V}-\mathrm{V}$ distances without variation of the $\mathrm{V}-\mathrm{O}$ distances. The structural modulation in $\mathrm{VOCl}$ causes thus less lattice-energy loss than for instance a direct bond-distance modulation.

The splitting of the diagonal $\mathrm{V}-\mathrm{V}$ distances is accompanied by a small splitting of the $\mathrm{V}-\mathrm{O}-\mathrm{V}$ angles leading to a smaller angle at the antiferromagnetic bond which seems to contradict the first estimation that the magnetic interaction should increase with the bond. However, in this $t_{2 g}$ system the direct overlap varied through the diagonal V-V distance seems to be the dominant effect.

\section{CONCLUSION}

In conclusion we have studied the nuclear and magnetic structures of VOCl. Synchrotron diffraction measurements reveal a structural phase transition $P m m n \rightarrow P 2 / n$ which accompanies the magnetic ordering in $\mathrm{VOCl}$. By neutron diffraction we were able to refine the magnetic structure within a monoclinic $2 \times 2 \times 2$ unit cell and derive a 3Dantiferromagnetic structure with an antiferromagnetic moment pointing in $a$ direction. At $2 \mathrm{~K}$ this moment amounts to about $1.3 \mu_{B}$ which is a significantly reduced value for a $\mathrm{V}^{3+}$ ion in this $3 d^{2}$ system.

Magnetoelastic coupling plays an important role in $\mathrm{VOCl}$ due to the fact that the magnetic exchange interaction depends sensitively on the V-O-V bond angles and on the orbital overlap. The modulation of the bond angles and of $\mathrm{V}-\mathrm{V}$ distances involves less lattice-energy cost than, e.g., bondlength modulation, so that rather strong structural effects occur. First the VO layers flatten upon cooling which can be well understood in terms of an enhanced magnetic interaction at low temperatures. The anisotropic thermal expansion further strengthens the enhanced magnetic interaction at low temperature. Second, the monoclinic distortion accompanying the magnetic ordering lifts the frustration of the coupling between two magnetic subsets as the diagonal V-V distances shrink in one diagonal direction enhancing the orbital overlap and, thus, the exchange interaction.

\section{ACKNOWLEDGMENTS}

This work was supported by the Deutsche Forschungsgemeinschaft through Sonderforschungsbereich 608 .

\footnotetext{
*braden@ph2.uni-koeln.de

${ }^{1}$ M. Shaz, S. van Smaalen, L. Palatinus, M. Hoinkis, M. Klemm, S. Horn, and R. Claessen, Phys. Rev. B 71, 100405(R) (2005).

${ }^{2}$ A. Seidel, C. A. Marianetti, F. C. Chou, G. Ceder, and P. A. Lee, Phys. Rev. B 67, 020405(R) (2003).
}

\footnotetext{
${ }^{3}$ V. Kataev, J. Baier, A. Möller, L. Jongen, G. Meyer, and A Freimuth, Phys. Rev. B 68, 140405(R) (2003).

${ }^{4}$ R. Rückamp, J. Baier, M. Kriener, M. W. Haverkort, T. Lorenz, G. S. Uhrig, L. Jongen, A. Möller, G. Meyer, and M. Grüninger, Phys. Rev. Lett. 95, 097203 (2005).
} 
${ }^{5}$ G. Caimi, L. Degiorgi, N. N. Kovaleva, P. Lemmens, and F. C. Chou, Phys. Rev. B 69, 125108 (2004).

${ }^{6}$ A. Schönleber, S. van Smaalen, and L. Palatinus, Phys. Rev. B 73, 214410 (2006).

${ }^{7}$ M. Hase, I. Terasaki, and K. Uchinokura, Phys. Rev. Lett. 70, 3651 (1993).

${ }^{8}$ J. P. Vénien, P. Palvadeau, D. Schleich, and J. Rouxel, Mater. Res. Bull. 14, 891 (1979).

${ }^{9}$ A. Wiedenmann, J. P. Venien, P. Palvadeau, and J. RossatMignod, J. Phys. C 16, 5339 (1983).

${ }^{10}$ S. M. Kauzlarich, J. L. Stanton, J. Faber, Jr., and B. A. Averill, J. Am. Chem. Soc. 108, 7946 (1986).

${ }^{11}$ S. R. Hwang, W.-H. Li, K. C. Lee, J. W. Lynn, and C.-G. Wu, Phys. Rev. B 62, 14157 (2000).

${ }^{12}$ A. N. Christensen, T. Johansson, and S. Quézel, Acta Chem. Scand., Ser. A 28, 1171 (1975).

${ }^{13}$ A. Haase and G. Brauer, Acta Crystallogr., Sect. B: Struct. Crys- tallogr. Cryst. Chem. 31, 2521 (1975).

${ }^{14}$ E. Benckiser, R. Rückamp, T. Möller, T. Taetz, A. Möller, A. A. Nugroho, T. T. M. Palstra, G. S. Uhrig, and M. Grüninger, New J. Phys. 10, 053027 (2008).

${ }^{15}$ M. Braden, G. Wilkendorf, J. Lorenzana, M. Aïn, G. J. McIntyre, M. Behruzi, G. Heger, G. Dhalenne, and A. Revcolevschi, Phys. Rev. B 54, 1105 (1996).

${ }^{16}$ W. Geertsma and D. Khomskii, Phys. Rev. B 54, 3011 (1996); R. Werner, C. Gros, and M. Braden, ibid. 59, 14356 (1999).

${ }^{17}$ H. Schäfer and F. Wartenpfuhl, J. Less-Common Met. 3, 29 (1961).

${ }^{18}$ M. Knapp, C. Baehtz, H. Ehrenberg, and H. Fuess, J. Synchrotron Radiat. 11, 328 (2004).

${ }^{19}$ M. Knapp, V. Joco, C. Baehtz, H. H. Brecht, A. Berghaeuser, H. Ehrenberg, H. von Seggern, and H. Fuess, Nucl. Instrum. Methods Phys. Res. A 521, 565 (2004).

${ }^{20}$ J. Rodriguez-Carvajal, Physica B 192, 55 (1993). 